\title{
Disability \\ considerations \\ during \\ the COVID-19 \\ outbreak
}

COVID-19

www.who.int/

emergencies/

diseases/novel-

coronavirus-2019

NCDs and mental health

www.who.int/ncds

www.who.int/mental_ health

Disability

www.who.int/healthtopics/disability
On 30 January 2020, the World Health Organization (WHO) declared the outbreak of a novel coronavirus disease, COVID-19, to be a Public Health Emergency of International Concern (PHEIC), due to the speed and scale of transmission.

WHO and public health authorities around the world are taking action to contain the COVID-19 outbreak. ${ }^{1}$ Certain populations, such as those with disability, may be impacted more significantly by COVID-19. This impact can be mitigated if appropriate actions and protective measures are taken by key stakeholders. 


\section{COVID-19}

\section{Why are additional considerations needed for people with disability during the COVID-19 outbreak?}

Actions need to be taken to ensure that people with disability can always access the health-care services, water and sanitation services and public health information they require, including during the COVID-19 outbreak.

People with disability may be at greater risk of contracting COVID-19 because of:

- Barriers to implementing basic hygiene measures, such as handwashing (e.g. handbasins, sinks or water pumps may be physically inaccessible, or a person may have physical difficulty rubbing their hands together thoroughly);

- Difficulty in enacting social distancing because of additional support needs or because they are institutionalized;

- The need to touch things to obtain information from the environment or for physical support;

- Barriers to accessing public health information.

People with disability may be at greater risk of developing severe disease if they become infected because of:

- The pre-existing health condition underlying the disability; and

- Barriers to accessing health care.

People with disability may also be disproportionately impacted by the outbreak because of serious disruptions to the services they rely on.

The barriers experienced by people with disability can be reduced if key stakeholders take appropriate action. 


\section{COVID-19}

\section{Considerations for actors}

\section{Actions for people with disability and their household}

\section{Reduce your potential exposure to COVID-19}

Everyone with disability and their household should follow the WHO guidance on basic protection measures during the COVID-19 outbreak, such as hand hygiene, respiratory etiquette and physical distancing. ${ }^{2}$ If you have any difficulty following these basic protection measures (for example, you are not able to access a handbasin/sink/water pump to wash your hands regularly), work with your family, friends and caregivers to identify adaptations. In addition

- Practice physical distancing of at least one meter from others.

- Avoid crowded environments to the maximum extent possible and minimize physical contact with other people. Consider making necessary visits to the supermarket or pharmacy, outside of peak time periods. Take advantage of special opening hours of stores for people with disability where these are offered.

- Make purchases online or over the telephone or request assistance from family, friends, or caregivers to avoid needing to access crowded environments.

- Consider buying in bulk items you need such as food, cleaning supplies, medication or medical supplies to reduce the frequency with which you need to access public places.

- Work from home if possible, especially if you typically work in a busy or crowded environment.

- Ensure that assistive products, if used, are disinfected frequently; these include wheelchairs, walking canes, walkers, transfer boards, white canes, or any other product that is frequently handled and used in public spaces.

\section{Put a plan in place to ensure continuation of the care and support you need}

- If you rely on caregivers, consider increasing the pool of those you can call upon, in preparation of one or more becoming unwell or needing to self-isolate. 


\section{COVID-19}

- If you organize caregivers through an agency, find out what contingency measures they have in place to compensate for a potential workforce shortage. You may want to talk to family and friends about what additional support they can provide, and the scenarios in which you may need to call upon them.

- Identify relevant organizations in your community that you can access if you need help.

\section{Prepare your household for the instance you should contract COVID-19}

- Make sure those in your household, including the friends and family you trust, know of any important information they would need should you become unwell. ${ }^{3}$ This may include information about your health insurance, your medication, and the care needs of any of your dependants (children, elderly parents or pets).

- Follow local advice regarding calling health care professionals or health care hotlines.

- Make sure everybody in your household knows what they should do should you contract COVID-19 or require assistance.

- If they are not already connected, introduce people in your support network so that they can communicate effectively should you become unwell.

- Know the telephone number of relevant telehealth ${ }^{4}$ services and hotlines, should you have questions or require non-urgent medical assistance.

\section{The mental and physical health of household members and caregivers}

- Ensure all members of the household and caregivers enact the basic protection measures, such as hand wash, against contracting COVID-19.

- Follow the WHO guidance on mental health considerations and guidance on managing existing noncommunicable diseases during the COVID-19 outbreak. ${ }^{5,6}$

- Encourage children with disability to continue playing, reading, learning, and connecting with friends using telephone calls, texts or social media.

3 https://apps.who.int/iris/bitstream/handle/10665/330987/WHO-nCov-IPC_Masks-2020.1-eng. pdf?sequence=1\&isAllowed=y

4 Telehealth involves the use of telecommunications and virtual technology to deliver health care outside of traditional health-care facilities

5 https://www.who.int/docs/default-source/coronaviruse/mental-health-considerations. pdf?sfvrsn=6d3578af_2

6 https://www.who.int/who-documents-detail/covid-19-and-ncds 


\section{COVID-19}

- If anyone in the household is suspected to have the virus, the person should be isolated, instructed to wear a mask, and must access testing as soon as possible. ${ }^{7}$ All surfaces need to be disinfected, and everyone in the household monitored for symptoms. If possible, anyone with an underlying health condition or reduced immunity needs to be moved to a separate location until the completion of isolation periods. 


\section{COVID-19}

\section{Actions for governments}

\section{Ensure public health information and communication is accessible}

- Include captioning and sign language for all live and recorded events and communications. This includes national addresses, press briefings, and live social media.

- Convert public materials into "Easy Read" format so that they are accessible for people with intellectual disability or cognitive impairment.

- Develop accessible written information products by using appropriate document formats, (such as "Word"), with structured headings, large print, braille versions and formats for people who are deafblind.

- Include captions for images used within documents or on social media. Use images that are inclusive and do not stigmatize disability.

- Work with disability organizations, including advocacy bodies and disability service providers to disseminate public health information.

\section{Undertake targeted measures for people with disability and their support networks}

Work with people with disability and their representative organizations to rapidly identify fiscal and administrative measures, such as:

- Financial compensation for families and caregivers who need to take time off work to care for loved ones. This could include paying, for a time-limited period, family members for support provided during normal working hours.

- Financial compensation for families and caregivers who are part of the casual and self-employed disability workforce, who may need to selfisolate, and where coming to work would place people with disability at greater risk of infection.

- Adoption of flexible, work-from-home policies, along with financial compensation for the technology required to do so.

- Financial measures (commonly within a broader-based economic stimulus package) that include people with disability, such as lump sum payments for qualifying individuals, tax relief, subsidization of items and/ or leniency and allowable deferral of common expenses.

- Appropriate action by schools and other educational facilities to ensure continued education for students with disability who may be required to study from home for longer periods. 


\section{COVID-19}

- Provision of a hotline in multiple formats (e.g. telephone and email) for people with disability to communicate with the government, ask questions, and raise concerns.

\section{Undertake targeted measures for disability service providers in the community ${ }^{8}$}

Work with the disability service providers to identify actions for the continuation of services and priority access to protective equipment:

- Ensure that agencies providing disability caregivers have continuity plans for situations in which the number of available caregivers may be reduced.

- Work with disability service providers to reduce bureaucratic recruitment barriers while still maintaining protection measures, such as police checks for caregivers.

- Consider short-term financial support for disability services to ensure they remain financially sustainable if they experience a downturn in their operations.

- Provide a hotline for disability services to communicate with government and raise concerns.

- Prioritize disability caregiver agencies for access to no-cost personal protective equipment, including masks, aprons, gloves and hand sanitizers.

- Ensure that caregivers of people with disability have access to COVID-19 testing alongside other identified priority groups.

\section{Increase attention given to people with disability living in potentially higher risk high-risk settings of developing the disease}

Work with people with disability and their representative organizations to identify actions to protect people with disability who may be in highrisk situations:

- Ensure that agencies providing services to people with disability in institutional settings ${ }^{9}$ develop and implement service continuity plans.

- Identify people with disability in prisons, jails and correctional facilities, and work with relevant authorities to implement infection control measures and identify possible contingencies.

8 Disability service providers in the community may include agencies that provide caregiver services, specialized employment opportunities, or specialized therapies and consultation to people with disability.

9 Institutional settings include prisons, psychiatric hospitals and care homes. 


\section{COVID-19}

- Ensure that people with disability who are homeless are provided with water, food, shelter and health care on an equal basis with others and are able to exercise basic protective measures against contracting COVID-19.

- Ensure that the needs of people with disability are considered in readiness and response operations for the COVID-19 outbreak in humanitarian settings, including those living in situations of forced displacement, in refugee or migrant camps, informal settlements and urban slums.

\section{Ensure that emergency measures include the needs of people with disability}

Work with people with disability and their representative organizations to ensure that emergency declarations based on the COVID-19 outbreak include their needs:

- Ensure that disability caregivers are considered as essential workers and exempted from curfews and other lockdown measures that may affect the continued provision of support services.

- Grant exemptions, so that people with disability who may experience significant distress with home confinement are permitted to leave their homes for short periods and in a safe way during curfews and other lockdown measures.

- Ensure that emergency measures do not discriminate on the basis of disability. Human rights protection mechanisms for people with disability placed in institutional settings should not be reduced as part of emergency measures. 


\section{COVID-19}

\section{Actions for health-care}

\section{Ensure COVID-19 health care is Accessible, Affordable and Inclusive}

- Work to ensure all clinics providing testing and services related to COVID-19 are completely accessible. Address physical barriers (such as uneven pathways, stairs, hard-to-reach spaces or hard-to-use equipment); attitudinal barriers (such as social stigma against disability and the denial of essential services); and financial barriers (such as high costs related to treatment or accessing the facility). Ensure that information about the accessibility of COVID-19 health services is disseminated to people with disability and their caregivers.

- Deliver information in understandable and diverse formats to suit different needs. Do not rely solely on either verbal or written information, and adopt ways to communicate that are understandable to people with intellectual, cognitive and psychosocial impairments.

- Deliver home-based consultations for people with disability, including for their general health needs and, where appropriate, for COVID-19 related needs.

- Develop and disseminate information to health workers so that they are aware of the potential health and social consequences of COVID-19 for people with disability.

- Deliver sufficient support for people with disability with more complex needs, particularly if quarantined or isolated. When needed, coordinate care between health and social services, families, and caregivers.

- Ensure that decisions on the allocation of scarce resources (e.g. ventilators) are not based on pre-existing impairments, high support needs, quality of life assessments, or medical bias against people with disability. Follow WHO guidance to prioritize those at high risk. ${ }^{10}$

\section{Deliver telehealth for people with disability}

- Provide telephone consultations, text messaging and video conferencing for the delivery of health care and psychosocial support for people with disability. This may be for their general health, and include rehabilitation needs and, where appropriate, COVID-19 related needs. 


\section{COVID-19}

\section{Actions for disability service providers in the community}

\section{Develop and implement service continuity plans}

- Plan for a scenario in which the workforce is reduced, and identify actions for scaling up administration and technical staff, as well as caregivers, where appropriate.

- Identify actions and work with government to reduce recruitment barriers, while continuing to maintain protection measures, such as police checks for caregivers.

- Hold additional trainings and if possible, develop online modules to prepare a new workforce and those who will take on expanded roles.

- Work with other local disability and caregiver agencies to undertake the prioritization of the most critically needed disability services and those that are essential to be kept open. Identify the clients most vulnerable to a reduction in services.

\section{Communicate frequently with people with disability and their support networks}

- Provide additional targeted information on COVID-19, highlighting information relevant to people with disability and their support networks. This may include information on continuity plans; telehealth and hotline phone numbers; locations of accessible health services; and locations where hand sanitizer or sterilizing equipment can be accessed when their supplies are low, or in situations where they may be required to self-isolate.

- Use a variety of communication platforms such phone calls, text and social media to share information, and convert existing information to accessible formats where necessary.

\section{Reduce potential exposure to COVID-19 during provision of disability services in the community}

- Provide training, and rapidly upskill the disability care workforce regarding infection control. ${ }^{11}$

- Ensure that disability caregivers and service providers have access to personal protective equipment including masks, gloves and hand sanitizers; consider increasing orders of these products. ${ }^{12}$

11 https://www.who.int/publications-detail/infection-prevention-and-control-during-health-carewhen-novel-coronavirus-(ncov)-infection-is-suspected-20200125

12 https://apps.who.int/iris/bitstream/handle/10665/331695/WHO-2019-nCov-IPC_PPE_use-2020.3eng.pdf 


\section{COVID-19}

- Deliver appropriate disability services through home-based consultation or through similar platforms as used in telehealth.

Provide sufficient support for people with disability who have complex needs

- Identify people with disability with more complex needs, and work with them, their families, and community support agencies, to identify contingencies for when the number of caregivers is limited or there are none available.

- Identify the potential for increased violence, abuse and neglect against people with disability because of social isolation and disruption to daily routines; support mitigation of these risks, for example providing an accessible hotline to report. 


\section{COVID-19}

\section{Actions for institutional settings}

\section{Reduce potential exposure to COVID-19}

Undertake immediate action to reduce potential exposure to COVID-19 in institutional settings ${ }^{13}$ :

- Identify those most at risk and work with them, their families and staff to implement infection control measures.

- Ensure that facilities are clean and hygienic, and that sanitation, washing facilities and supplies are available and accessible.

- Reduce crowding to the maximum extent possible by modifying the distribution of spaces.

- Reduce the number of people in psychiatric hospitals, wherever possible, by implementing schemes of early discharge, together with provision of adequate support for living in the community.

- Ensure that residents can access information about COVID-19 and know how to follow the basic protection measures. ${ }^{14}$

- Implement protective measures to prevent the spread of infections during visiting hours, and facilitate different methods of communication with families and the outside world (e.g. telephone, internet, video communication).

\section{Prepare for COVID19 infections in institutions ${ }^{15}$}

- Provide testing and medical care to residents when needed; refer residents to appropriate medical facilities as necessary.

- Prepare for an increased need of support staff to care for those who contract mild cases of COVID-19 but do not need hospitalization.

- Provide appropriate personal protective equipment when needed to residents with disability and staff.

- Implement infection control measures for residents with COVID-19 who do not need hospitalization (e.g. instruct them to wear a mask and limit contact with other residents).

\section{Provide sufficient support for residents with disability}

- Ensure that sufficient staff and supplies are maintained to safeguard the continuation of care and support for residents. 


\section{COVID-19}

- Provide access to psychosocial support for residents experiencing distress during the outbreak, including through call-in and online psychosocial services and peer support.

\section{Guarantee the rights of residents during the COVID-19 outbreak}

- Check and guarantee that residents are not being abused or neglected and that coercive measures are not being used or escalated during the outbreak.

- Ensure that the existing monitoring and complaints mechanisms remain functioning and effective. 


\section{COVID-19}

\section{Actions for the community}

\section{Basic protection measures to be adopted by the general public}

- Follow the guidance prepared by WHO on basic protection measures against COVID-19.16 Take the risk of COVID-19 seriously; even if you, yourself, may not be at high risk of serious symptoms, you may pass the virus on to someone that is.

\section{Flexible work arrangements and infection control measures to be supported by employers}

- Follow WHO guidance on getting your workplace ready for the COVID-19 outbreak. ${ }^{17}$

- Where possible, implement flexible working arrangements that allow people with disability to telework. Ensure they have the technology they need, including any assistive products typically available in the workplace.

- If teleworking is not possible, consider allowing people with disability at high risk of severe symptoms to take leave (including paid leave) until the risk of infection is reduced. Explore government policy and support that may be available to employers to enable the implementation of these measures.

- Ensure the accessibility of workplace infection control measures, such as hand sanitization stations.

\section{Increased access to stores to be provided by store owners for vulnerable populations}

- Consider providing allocated hours for people with disability or other potentially vulnerable people to access the store; or consider alternative ways to allow people with disability to shop (e.g. delivery, online).

WHO reference number: WHO/2019nCoV/Disability/2020.1

(c) World Health Organization 2020. Some rights reserved. This work is available under the CC BY-NCSA 3.0 IGO licence.

\section{Extra support to be provided by family, friends and neighbours for a person with disability}

- Check in regularly with a person with disability to provide emotional and practical support, respecting social isolation restrictions that may be in place.

- Know the facts, be informed and don't spread rumors related to COVID-19. ${ }^{18}$

16 https://www.who.int/emergencies/diseases/novel-coronavirus-2019/advice-for-public

17 https://www.who.int/docs/default-source/coronaviruse/getting-workplace-ready-for-covid-19. pdf?sfvrsn=359a81e7_6

18 https://www.who.int/emergencies/diseases/novel-coronavirus-2019/advice-for-public/mythbusters 